\title{
EFECTOS DEL CAPITAL HUMANO EN LAS ORGANIZACIONES EMPRESARIALES: ESTIMACIONES ECONOMÉTRICAS PARA NUEVOS NEGOCIOS Y EXPORTACIONES
}

\author{
HUMAN CAPITAL EFFECTS ON BUSINESS \\ ORGANIZATIONS: ECONOMETRIC ESTIMATES FOR \\ NEW BUSINESSES AND EXPORTS
}

Recepción: 2019-11-04 - Aceptación: 2020-05-16

\author{
Marlit Marín Hernández ${ }^{1 ;}$ a \\ (iD) ORCID iD 0000-0001-5310-248X \\ Edwin Arbey Hernández García ${ }^{1 ; ~ b ~}$ \\ (iD) ORCID iD 0000-0002-5919-7659 \\ ${ }^{1}$ Universidad del Valle, Cali, Colombia. \\ a Magíster en Administración. \\ b Magíster en Economía Aplicada.
}

\section{RESUMEN}

Objetivo: mostrar el impacto positivo del capital humano sobre la generación de nuevos negocios y el nivel de exportaciones. Método: se hace una revisión de literatura y luego empíricamente se estiman dos modelos de datos panel para medir el impacto del capital humano sobre variables económicas empresariales. Resultados: se encontró que la variable de gasto público en educación tiene efecto marginal positivo sobre la generación de nuevos negocios y las exportaciones. Por su parte, la inversión en educación secundaria tiene efectos positivos y significativos sobre nuevos negocios empresariales, siendo esta época de formación educativa, propicia para generar un espíritu emprendedor en los jóvenes. Por su parte, todas las variables de educación tuvieron impactos positivos y significativos sobre las exportaciones. Conclusiones: se pueden encontrar efectos positivos del capital humano, especialmente de la educación, sobre la generación de nuevos negocios y sobre las exportaciones.

Palabras clave: Capital humano; Organizaciones empresariales; Nuevos negocios; Exportaciones; Datos panel.

\begin{abstract}
Objective: to show the positive impact of human capital about new businesses and the level of exports. Method: a literature review is done and after empirically two panel data models are estimated to measure the impact of human capital on business economic variables. Results: It was found that the public spending on education has a positive marginal effect on the generation of new businesses and the exports. For its part, investment in secondary education has positive and significant effects on new business ventures, being secondary education appropriate to generating an entrepreneurial behavior in young people. For their part, all education variables had positive and significant impacts on exports. Conclusions: it could be possible to find positive effects of human capital, especially in education, about the creation of new businesses and over exports.
\end{abstract}

Keywords: Human capital; Business organizations; New businesses; Exports; Data panel. 


\section{INTRODUCCIÓN}

La génesis del capital humano como objeto de estudio apareció para finales de la década de los años cincuenta e inicios de los sesenta del siglo XX por Mincer (1958), Schultz (1961) y Becker (1962). En este inicio del marco teórico, se definió el capital humano como la acumulación de conocimientos, experiencias y habilidades que propende por incrementar la productividad y por lo tanto los ingresos de las organizaciones empresariales y el retorno de los individuos. De esta manera las inversiones en el bienestar individual, la capacitación, los niveles de escolarización y las experiencias laborales, son útiles para mejorar la productividad y así mismo los ingresos. La teoría ha avanzado con múltiples investigaciones que señalan el capital humano como relevante para explicar el desarrollo económico de las naciones, las empresas y las mejoras del bienestar individual.

En lo que se refiere a la evolución que ha tenido esta teoría, se han conocido varios contextos históricos que han ido formando sistemas productivos y relaciones sociales interesantes para la sociedad. Un ejemplo muy particular es el paso del sistema agrícola tradicional a la industrialización, y luego su paso a la post-industrialización (Bell, 1973). La principal característica de la sociedad postindustrial, según Bell, es la codificación del conocimiento teórico y la nueva relación entre ciencia y tecnología. Los principales desarrollos postindustriales son: el aumento del sector servicios, los cambios ocupacionales, los cambios en las profesiones y la educación, el creciente papel del capital financiero y humano, las nuevas formas de infraestructura y la teoría del valor del conocimiento.

Hay autores que a través de la historia han explicado cómo se relaciona la mano de obra, sus niveles de educación y la influencia sobre la productividad empresarial. En el caso de Smith (2009), la división social del trabajo causaba mayores niveles de educación y preparación en los individuos, que con la aplicación de dichos conocimientos en la industria llevaría a mayores niveles de producción. Por su parte Say (2001) consideraba que los empresarios debían darle la importancia merecida a la adquisición de conocimientos, desarrollar destrezas y habilidades en las actividades realizadas para aumentar la productividad. Por su parte Stuart-Mill (2008) pensaba que mejoras en la educación y las destrezas laborales generaban impactos positivos sobre la producción empresarial a través de la capacidad para maniobrar máquinas más complejas y a la capacidad de innovar en la generación de nuevas herramientas y formas de trabajo.

Para esta etapa clásica de Smith, Say y Stuart-Mill, en general el planteamiento fue que primero se constituyeron las formas de producción y luego el conocimiento para mejorar y perfeccionar dichas formas o el arte de hacer el trabajo. Por eso es importante mencionar que el capital humano representado para la época en los niveles de preparación o educación, no fue considerado abiertamente como un motor del crecimiento económico o de las formas de producción. Solo a partir de finales de los años 50 del siglo XX, cuando las formas de producción, la industria y las empresas se encontraron en etapas superiores del desarrollo, se comenzó a considerar el capital humano como parte fundamental del crecimiento económico de los países, de las organizaciones y de los individuos, dándose el inicio formal de lo que hoy en día se conoce como la teoría del capital humano.

En este sentido, autores como Goldin y Katz (1999), escribieron que la demanda por trabajadores altamente cualificados y buenas habilidades laborales, llevaron al cambio de producción en el siglo XX, con una sociedad moderna y de avances tecnológicos significativos. Precisamente en dicho siglo es que autores como Castells (2000) comentaron que fue un periodo donde la sociedad y las empresas encontraron en el capital humano y el capital intelectual, mediante el uso de la información y el conocimiento, la nueva fuente de generación de riqueza.

Adicionalmente, otros aspectos que han estimulado el capital humano e intelectual para mejorar la producción y obtener mayor valor agregado, han sido: (i) la gestión del conocimiento, (ii) la potencialización creativa del individuo como resultado de las competencias desarrolladas gracias a acciones como capacitaciones, mentoring y coaching, (iii) el manejo de un orden flexible en las áreas de especialización como las tareas repetitivas, (iv) la fundamentación de una cultura y un clima organizacional donde se fortalecen valores como la innovación, la creatividad, el aprendizaje y se motivan a vivir nuevas experiencias basadas en el conocimiento constante, (v) la motivación al personal para realizar investigaciones con las cuales se logren generar cambios constantemente (Alles, 2005; Castillo, 2012).

Dichos aspectos relacionados con las mejoras del talento humano, capital intelectual y gestión del conocimiento, como parte esencial de lo que es el desarrollo del capital humano, generan impactos positivos e importantes sobre la producción, incremento de las ventas, fluidez en los procesos administrativos y jurídicos al interior de las organizaciones empresariales. Cada vez más se reconoce que el conocimiento y su aplicación intensiva, así como la salud mental y física de los individuos, son elementos primordiales y fuente de creación y generación de valor, riqueza y ventaja competitiva en las organizaciones (Bueno, Salmador y Merino, 2008). 
Así es como diversos estudios han mostrado que existe una alta correlación positiva entre las variables del capital humano y variables empresariales como la producción, la innovación, la adopción de nuevas tecnologías, la competitividad, el valor de las acciones (Warech y Tracey, 2004; Pulic, 2004; Fitz-enz, 2009; Marín, Hernández y Burbano, 2018), entre otras. Así mismo, hay correlaciones altas entre cada una de estas variables empresariales, de forma tal que, por ejemplo, mejoras en la educación y la experiencia por parte de las personas conllevan adopciones más rápidas de las nuevas tecnologías que incentivan la innovación y por lo tanto incrementos en la productividad y la competitividad (Nelson y Phelps, 1996; Mungaray y Ramírez, 2007).

Por el lado de variables del comercio internacional, estudios han mostrado que el capital humano tiene efectos positivos sobre las exportaciones y la internacionalización de las empresas, entre otros aspectos, se encuentran el dominio de idiomas, las reglas de negociación internacional, los contactos de pares internacionales, una actividad positiva hacia las exportaciones, habilidades para resolver problemas y de negociación con personas de otras culturas.

A nivel teórico autores como Barney (1991) y Peteraf (1993) comentan que los recursos humanos como parte de las capacidades internas de una organización y origen importante de sus ventajas competitivas, generan rendimientos positivos sobre las empresas exportadoras. A nivel empírico trabajos como los de Manolova, Brush, Edelman y Greene (2002) en empresas pequeñas de los Estados Unidos, López (2006) para la empresa manufacturera española, Andersson y Johansson (2010) en empresas industriales de Suecia, Pucar (2012) para empresas de Bosnia, y Castillo (2017) para empresas de los sectores textil y metalmecánica en Perú, demuestran la influencia positiva del capital humano sobre la internacionalización y las exportaciones de las organizaciones empresariales.

Dados los planteamientos teóricos anteriores, la pregunta problema que dirige esta investigación es: ¿cómo impacta el capital humano las organizaciones empresariales al nivel de sus variables como generación de nuevos negocios y nivel de exportaciones? Siendo así un trabajo empírico que se basa metodológicamente en econometría de datos panel para sus estimaciones.

Por lo cual la hipótesis es: el capital humano tiene efectos positivos sobre las organizaciones empresariales, específicamente sobre la generación de nuevos negocios y sobre las exportaciones que llevan a cabo las empresas hacia el resto del mundo.

El objetivo general de este artículo es presentar el impacto del capital humano sobre las organizaciones empresariales en los aspectos de nuevos negocios y de exportaciones. En este sentido, los objetivos específicos son: en primer lugar, estimar econométricamente los impactos del capital humano sobre los nuevos negocios; $y$ en segundo lugar, estimar econométricamente los impactos del capital humano sobre las exportaciones. Estos objetivos permitirán poner a prueba el efecto del capital humano sobre las organizaciones a nivel de nuevos negocios y nivel de exportaciones empresariales.

\section{MATERIAL Y MÉTODOS}

Con el fin de estimar los impactos del capital humano sobre variables empresariales, se presentan a continuación las ecuaciones econométricas. Para analizar los impactos sobre las organizaciones empresariales, se han escogido dos: generación de nuevos negocios y exportaciones. Tanto las variables dependientes como de controles han sido seleccionadas de los indicadores del Banco Mundial, en el periodo 1980-2017 y un máximo de 112 países en el mundo.

En primer lugar, se presenta la ecuación de tipo panel que relaciona la creación de negocios, las variables relacionadas con el capital humano y las de controles:

$$
\ln \left(N e g_{i t}\right)=\alpha_{0}+\alpha_{1} \ln \left(Y_{i t}\right)+\alpha_{2} \ln \left(\frac{K_{i}}{L_{i}}\right)_{t}+\alpha_{3} \ln \left(\frac{T_{i}}{L_{i}}\right)_{t}+\alpha_{4} \ln \left(\mathrm{CH}_{i t}\right)+\varepsilon_{i t}
$$

Donde $\ln \left(\mathrm{Neg}_{i t}\right)$ es la variable dependiente en logaritmo natural que mide la cantidad de nuevos negocios registrados en el año calendario para cada país $i$ en el periodo $t$. La variable $\ln \left(Y_{i t}\right)$ corresponde al PIB real en dólares constantes de 2010 de cada país $i$ en el periodo $t$. Por su parte la relación capital-trabajador $\left(\mathrm{K}_{\mathrm{i}} / \mathrm{L}_{\mathrm{i}}\right)_{\mathrm{t}}$ fue estimada bajo la metodología de Lora (1994) y para su construcción se utilizaron las estadísticas del Banco Mundial. La relación $\left(T_{i} / L_{i}\right)$ se construyó también teniendo en cuenta los indicadores del Banco Mundial. En el caso de la variable $\ln \left(\mathrm{CH}_{i t}\right)$ representa cada uno de los indicadores que se van a usar para medir el impacto de la educación y la salud sobre la creación de nuevas empresas. $\varepsilon_{i t}$ es el error estocástico que se distribuye 
independiente e idénticamente con $\mathrm{E}\left(\varepsilon_{i t}\right)=0$ y $\operatorname{Var}\left(\varepsilon_{i t}\right)=\sigma^{2}>0$.

Como la ecuación (1) representa una estructura de datos panel, las estimaciones econométricas se llevaron a cabo bajo el estimador de efectos fijos corregidos por autocorrelación y heterocedasticidad por medio de los errores estándar corregidos para panel -PCSE- teniendo en cuenta que en cada uno de los modelos se presentan problemas de autocorrelación y heterocedasticidad, pruebas que están al final de la Tabla 1 (Beck y Katz, 1995; Beck, 2001).

En segundo lugar, la ecuación (2) permite estimar el efecto del capital humano sobre las exportaciones de bienes y servicios, y se expresa como sigue:

$$
\ln \left(X_{i t}\right)=\alpha_{0}+\alpha_{1} \ln \left(Y_{i t}\right)+\alpha_{2} \ln \left(\frac{K_{i}}{L_{i}}\right)_{t}+\alpha_{3} \ln \left(\frac{T_{i}}{L_{i}}\right)_{t}+\alpha_{4} \ln \left(C H_{i t}\right)+\varepsilon_{i t}
$$

Donde $\ln \left(X_{i t}\right)$ son las exportaciones en logaritmo natural para cada país $i$ en el periodo $t$. Las demás variables independientes son las mismas de la ecuación (1) y que se mencionaron antes. Así mismo, la ecuación (2) representa una estructura de datos panel, por lo cual las estimaciones econométricas se han hecho bajo el estimador PCSE (Beck y Katz, 1995; Beck, 2001).

\section{RESULTADOS}

La siguiente Tabla 1 muestra las estimaciones de la ecuación número (1), evidenciando que la variable del capital humano relacionada con el gasto público en educación, tiene un efecto marginal positivo sobre la generación

Tabla 1

Impactos del capital humano sobre nuevos negocios en el periodo 2005-2017

\begin{tabular}{|c|c|c|c|c|c|}
\hline Variables explicativas & Modelo 1 & Modelo 2 & Modelo 3 & Modelo 4 & Modelo 5 \\
\hline PIB en dólares constantes base 2010 & $\begin{array}{c}0,590^{* * *} \\
(0,114)\end{array}$ & $\begin{array}{r}0,662^{* * *} \\
(0,038)\end{array}$ & $\begin{array}{l}0,629^{* * *} \\
(0,049)\end{array}$ & $\begin{array}{c}0,862^{* * *} \\
(0,101)\end{array}$ & $\begin{array}{c}0,806^{* * *} \\
(0,166)\end{array}$ \\
\hline $\begin{array}{l}\text { Capital por trabajador a precios constantes } \\
\text { base } 2010\end{array}$ & $\begin{array}{l}-0,054 \\
(0,048)\end{array}$ & $\begin{array}{l}-0,030 \\
(0,059)\end{array}$ & $\begin{array}{l}0,206^{\star * *} \\
(0,065)\end{array}$ & $\begin{array}{l}-0,054 \\
(0,177)\end{array}$ & $\begin{array}{l}-0,045 \\
(0,056)\end{array}$ \\
\hline $\begin{array}{l}\text { Tierra cultivable por trabajador en metros } \\
\text { cuadrados }\end{array}$ & $\begin{array}{c}0,001 \\
(0,031)\end{array}$ & $\begin{array}{l}0,076^{* *} \\
(0,034)\end{array}$ & $\begin{array}{c}0,001 \\
(0,046)\end{array}$ & $\begin{array}{c}0,352^{\star * *} \\
(0,113)\end{array}$ & $\begin{array}{c}0,021 \\
(0,031)\end{array}$ \\
\hline $\begin{array}{l}\text { Gasto público en educación en dólares } \\
\text { constantes de } 2010\end{array}$ & $\begin{array}{l}0,077 \\
(0,116)\end{array}$ & & & & \\
\hline $\begin{array}{l}\text { Gasto público por estudiante de educación } \\
\text { terciaria en dólares constantes de } 2010\end{array}$ & & $\begin{array}{c}0,029 \\
(0,048)\end{array}$ & & & \\
\hline $\begin{array}{l}\text { Gasto público por estudiante de educación } \\
\text { secundaria en dólares constantes de } 2010\end{array}$ & & & $\begin{array}{l}0,159^{* * *} \\
(0,049)\end{array}$ & & \\
\hline $\begin{array}{l}\text { Formación en Capital Humano (salud y } \\
\text { educación) }\end{array}$ & & & & $\begin{array}{c}0,330^{* * *} \\
(0,113)\end{array}$ & \\
\hline Gasto en salud en dólares constantes de 2010 & & & & & $\begin{array}{l}-0,128 \\
(0,149)\end{array}$ \\
\hline Constante & $\begin{array}{r}-7,21^{* * *} \\
(1,232)\end{array}$ & $\begin{array}{c}-7,69^{* * *} \\
(0,974)\end{array}$ & $\begin{array}{l}-6,96^{* * *} \\
(1,312)\end{array}$ & $\begin{array}{c}-14,3^{* * *} \\
(2,738)\end{array}$ & $\begin{array}{l}-8,1^{* * *} \\
(1,481)\end{array}$ \\
\hline R-cuadrado & 0,91 & 0,91 & 0,92 & 0,85 & 0,91 \\
\hline Observaciones & 925 & 817 & 831 & 229 & 961 \\
\hline \multicolumn{6}{|l|}{ Pruebas de elección } \\
\hline Prueba de Breusch-Pagan & $3999^{* * *}$ & $3545^{\star \star \star}$ & $3384^{* * *}$ & $442^{* \star \star}$ & $4267^{* * *}$ \\
\hline Prueba F & $127^{* * *}$ & $104^{* * *}$ & $105^{\star * *}$ & $29^{* * *}$ & $135^{* * *}$ \\
\hline Prueba de Hausman & $23^{* * *}$ & $33^{* * *}$ & $62^{* * *}$ & $18^{* * *}$ & $42^{* * *}$ \\
\hline \multicolumn{6}{|l|}{ Probando los supuestos del error } \\
\hline Prueba de heterocedasticidad & $79501^{* * *}$ & $47511^{* * *}$ & $74573^{* * *}$ & $2005^{* * *}$ & $75239^{* * *}$ \\
\hline Prueba de Autocorrelación & $23^{* * *}$ & $19^{* * *}$ & $16^{* * *}$ & $7^{* *}$ & $22^{* * *}$ \\
\hline Método de estimación & & & PCSE & & \\
\hline
\end{tabular}

Errores estándar entre paréntesis. Significativo al: $1 \%^{* * *} ; 5 \%{ }^{* *} ; 10 \%^{*}$

Fuente: cálculos propios con base en datos del Banco Mundial. 
de nuevos negocios, y solamente significativo para la inversión pública en educación de tipo secundaria, con lo cual se puede llegar a pensar que mayores políticas económicas de inversión sobre la educación secundaria y su mejora en calidad, puede incidir positivamente y de forma significativa en el desarrollo de nuevos negocios empresariales, siendo esta época de formación educativa propicia para generar y llevar a cabo el emprendimiento de jóvenes, con ideas nuevas y fuerzas que saquen adelante sus negocios. Y aunque según estas estimaciones, la inversión en educación pública terciaria para los países en su conjunto, no resultan significativas, el efecto marginal positivo esperado sí se cumple.

Por su parte, la otra variable del capital humano que resulta significativa y de efecto positivo sobre la creación de negocios es aquella nombrada como Formación en Capital Humano (salud y educación), que por la forma como están construidos los datos en el Banco Mundial, hace referencia a la calificación de formación de recursos humanos de las políticas e instituciones nacionales (públicas y privadas) que afectan el acceso a los servicios de salud y educación, como también su calidad, y los cuales se encuentran disponibles para 31 economías, razón por la cual el número de observaciones para el modelo 4 es menor que los demás.

Respecto al gasto en salud, los datos no muestran mayor contundencia sobre el impacto de dicha variable en creación de nuevos negocios, probablemente porque el impacto de la salud sobre las organizaciones empresariales se da más sobre la productividad misma de procesos contemporáneos, que sobre nuevos negocios o nuevos procesos productivos, de los cuales estos últimos necesitan más conocimiento (educación, por ejemplo) que buena salud. Aunque, este análisis queda abierto para futuras investigaciones.

Tabla 2

Impactos del capital humano sobre las exportaciones en el periodo 1980-2017

\begin{tabular}{|c|c|c|c|c|c|}
\hline Variables explicativas & Modelo 1 & Modelo 2 & Modelo 3 & Modelo 4 & Modelo 5 \\
\hline PIB en dólares constantes base 2010 & $\begin{array}{l}0,843^{* * *} \\
(0,028)\end{array}$ & $\begin{array}{l}0,857^{* * *} \\
(0,014)\end{array}$ & $\begin{array}{l}0,816^{* * *} \\
(0,019)\end{array}$ & $\begin{array}{l}0,963^{* * *} \\
(0,035)\end{array}$ & $\begin{array}{l}0,863^{* * *} \\
(0,042)\end{array}$ \\
\hline $\begin{array}{l}\text { Capital por trabajador a precios constantes } \\
\text { base } 2010\end{array}$ & $\begin{array}{c}0,137^{* * *} \\
(0,019)\end{array}$ & $\begin{array}{c}0,112^{* * *} \\
(0,022)\end{array}$ & $\begin{array}{c}0,105^{* * *} \\
(0,037)\end{array}$ & $\begin{array}{l}0,247^{* * *} \\
(0,082)\end{array}$ & $\begin{array}{c}0,129^{* * *} \\
(0,019)\end{array}$ \\
\hline $\begin{array}{l}\text { Tierra cultivable por trabajador en metros } \\
\text { cuadrados }\end{array}$ & $\begin{array}{c}-0,104^{* * *} \\
(0,018)\end{array}$ & $\begin{array}{c}-0,090^{* * *} \\
(0,018)\end{array}$ & $\begin{array}{c}-0,136^{* * *} \\
(0,024)\end{array}$ & $\begin{array}{c}0,209^{* * *} \\
(0,07)\end{array}$ & $\begin{array}{c}-0,078^{* * *} \\
(0,018)\end{array}$ \\
\hline $\begin{array}{l}\text { Gasto público en educación en dólares } \\
\text { constantes de } 2010\end{array}$ & $\begin{array}{l}0,050^{* *} \\
(0,021)\end{array}$ & & & & \\
\hline $\begin{array}{l}\text { Gasto público por estudiante de educación } \\
\text { terciaria en dólares constantes de } 2010\end{array}$ & & $\begin{array}{c}0,126^{* * *} \\
(0,017)\end{array}$ & & & \\
\hline $\begin{array}{l}\text { Gasto público por estudiante de educación } \\
\text { secundaria en dólares constantes de } 2010\end{array}$ & & & $\begin{array}{l}0,082^{* * *} \\
(0,015)\end{array}$ & & \\
\hline $\begin{array}{l}\text { Formación en Capital Humano (salud y } \\
\text { educación) }\end{array}$ & & & & $\begin{array}{c}0,265^{* * *} \\
(0,086)\end{array}$ & \\
\hline Gasto en salud en dólares constantes de 2010 & & & & & $\begin{array}{c}0,039 \\
(0,034)\end{array}$ \\
\hline Constante & $\begin{array}{l}1,239^{* * *} \\
(0,441)\end{array}$ & $\begin{array}{l}1,232^{* * *} \\
(0,358)\end{array}$ & $\begin{array}{c}2,741^{* * *} \\
(0,423)\end{array}$ & $\begin{array}{c}-2,719^{* *} \\
(1088)\end{array}$ & $\begin{array}{l}0,985^{*} \\
(0,505)\end{array}$ \\
\hline R-cuadrado & 0,99 & 0,99 & 0,99 & 0,99 & 0,98 \\
\hline Observaciones & 3578 & 1833 & 2985 & 390 & 3728 \\
\hline \multicolumn{6}{|l|}{ Pruebas de elección } \\
\hline Prueba de Breusch-Pagan & $33705^{* * *}$ & $16065^{* * *}$ & $17463^{* * *}$ & $1542^{* * *}$ & $37084^{* * *}$ \\
\hline Prueba F & $110^{* * *}$ & $211^{* * *}$ & $56^{* * *}$ & $202^{* * *}$ & $120^{* * *}$ \\
\hline Prueba de Hausman & $1454^{* * *}$ & $62^{* * *}$ & $28^{* * *}$ & $26^{* * *}$ & $43^{* * *}$ \\
\hline \multicolumn{6}{|l|}{ Probando los supuestos del error } \\
\hline Prueba de heterocedasticidad & $33000^{* * *}$ & $130000^{* * *}$ & $100000^{* * *}$ & $140000^{* * *}$ & $270000^{* * *}$ \\
\hline Prueba de Autocorrelación & $547^{* * *}$ & $155^{\star * *}$ & $388^{* * *}$ & $27^{* * *}$ & $282^{\star \star *}$ \\
\hline
\end{tabular}

Errores estándar entre paréntesis. Significativo al: $1 \%{ }^{* * * ;} ; 5 \%$ **; $10 \%$ *

Fuente: cálculos propios con base en datos del Banco Mundial. 
Por su parte, la Tabla 2 muestra las estimaciones de la ecuación (2), las cuales muestran el impacto positivo y significativo sobre las exportaciones. Este resultado consistente en todos los modelos estimados, es importante porque prueba lo que en teoría se comentó antes, es decir, que aspectos relacionados con la educación como el dominio de idiomas, las reglas de negociación internacional, las habilidades para negociar con individuos de otras culturas, entre otras, inciden en las capacidades internas de las empresas exportadoras y se constituyen como importantes ventajas competitivas en el mercado.

\section{DISCUSIÓN}

La revisión de literatura que se llevó a cabo en la introducción, mostró de forma general que el capital humano tiene efectos positivos sobre las organizaciones empresariales y respectivas variables económicas, entre ellas se pueden anotar: incrementos en la producción y el valor agregado, la mejora en ingresos por ventas, fluidez en los procesos administrativos y jurídicos al interior de las mismas organizaciones, generación de innovación y rápida adopción y adaptación de las nuevas tecnologías, mejoras del capital intelectual que luego inciden en mejores posiciones de los indicadores financieros, y en general mejora en la competitividad.

Así mismo, el capital humano incide de forma positiva sobre la creación de nuevos negocios y empresas, efecto que se pudo probar empíricamente con datos del Banco Mundial y estimaciones econométricas de datos panel. El impacto marginal es positivo y significativo, especialmente la educación de secundaria, y aunque el gasto en educación general y educación terciaria no resultaron significativas, sí mostraron un impacto marginal positivo sobre la generación de nuevos negocios. En términos de la discusión, es interesante probar empíricamente la relación positiva entre educación y emprendimiento, temas que han sido abordados por diferentes investigadores como Ferrante (2005), Braunerhjelm, Acs, Audretsch y Carlsson (2010) y Siqueira (2007), este último quien evidencia altas probabilidades de emprender nuevos negocios para aquellos individuos con niveles superiores de educación.

Sin embargo, no se encontró evidencia favorable del efecto de la salud sobre la generación de nuevos negocios, lo cual se pudo observar porque las estimaciones no resultaron significativas y el signo esperado no fue consistente. Aunque no ha sido ampliamente explorada la relación entre salud y nuevos negocios o emprendimiento, sí lo ha sido la relación entre salud e ingresos en los individuos, especialmente mediada por las condiciones laborales de los trabajadores. En este sentido los trabajos de Cutler y Lleras (2006), Thomas y Frankenberg (2002), Andrade y Gómez (2008) y Matabanchoy (2012), aportan evidencia teórica y empírica a esta relación. Consideramos que esta investigación es limitada a analizar la relación entre salud y nuevos negocios, por lo cual no es posible inferir resultados más allá de los encontrados, sin embargo, esta limitante puede ser una oportunidad de investigación con mayor alcance en el futuro.

Por su parte y relacionado con el comercio internacional, la literatura expuesta y las estimaciones econométricas, muestran los impactos positivos y significativos de la educación sobre las exportaciones, de tal manera que esta clase de capital humano incide positivamente en la mejora de ingresos de las empresas exportadoras, pero además mejora su posición de internacionalización. Discusión y resultados en la misma dirección que a nivel teórico respaldan autores como Barney (1991), Peteraf (1993), y a nivel empírico Bosnia y Castillo (2017), Pucar (2012) y Andersson y Johansson (2010) Marín et al. (2018), quienes demuestran los efectos positivos del capital humano sobre los procesos de internacionalización e incremento de las exportaciones de parte de las empresas.

Conclusiones: se pudo encontrar mediante las estimaciones econométricas de datos panel que efectivamente el capital humano, especialmente lo relacionado con la educación, tiene impactos positivos y significativos sobre la generación de nuevos negocios empresariales, y sobre las exportaciones de las empresas. En este sentido, Los esfuerzos a nivel organizacional, a nivel individual y de política económica, serán provechosos sobre la inversión en capital humano, que tendrá efectos positivos en el desarrollo y crecimiento de las empresas. Aunque no es nueva esta conclusión, es paradójico que aún los países y organizaciones empresariales, sabiendo los efectos positivos del capital humano, no hacen el esfuerzo para que sus ciudadanos o empleados puedan prepararse, adquirir conocimientos, estudiar y desarrollar una mejor calidad de vida, que luego se traducirá en incrementos del ingreso nacional e individual de las compañías.

\section{REFERENCIAS BIBLIOGRÁFICAS}

Alles, M. (2005). Desarrollo del talento humano basado en competencias. Buenos Aires: Ediciones Granica.

Andersson, M., \& Johansson, S. (2010). Human capital and the structure of regional export flows. Technology in Society, 32(3), 230-240. 
Andrade, V., \& Gómez I. (2008). Salud laboral. Investigaciones realizadas en Colombia. Pensamiento Psicológico, 4(10), 9-25.

Becker, G. S. (1962). Investment in Human Capital: A Theoretical Analysis. The Journal of Economy, 70(5), part $2,9-49$.

Bell, D. (1973). The coming of post-indusrial society: a venture in social forecasting. New York: Basic Books.

Bueno, E., Salmador, M. P., \& Merino, C. (2008). Génesis, concepto y desarrollo del capital intelectual en la economía del conocimiento: una reflexión sobre el modelo intellectus y sus aplicaciones. Estudios de Economía Aplicada, 26(2), 43-63.

Braunerhjelm, P., Acs, Z. J., Audretsch, D. B., \& Carlsson, B. (2010). The missing link: knowledge diffusion and entrepreneurship in endogenous growth. Small Business Economics, 34,105-125.

Castells, M. (2000). La era de la información. Economía, Sociedad y Cultura. Vol.1 La Sociedad Red. Madrid: Allianza Editorial.

Castillo, R. (2012). Desarrollo del capital humano en las organizaciones. México D.F.: Red Tercer Milenio.

Castillo, C. (2017). Capital intelectual y exportación: un análisis desde la perspectiva del conocimiento y del contexto. (Tesis de Doctorado, Departamento de Ingeniería, Sección de Organización de Empresas). Universidad Carlos III de Madrid, Madrid. Recuperado de https://e-archivo.uc3m.es/bitstream/handle/10016/25507/ Tesis_Carlos_delCastillo_Guardamino_2017.pdf?sequence=1

Cutler, D., \& Lleras, A. (2006). Education and Health: Evaluating Theories and Evidence. Working Paper No. 12352, National Bureau of Economic Research. Recuperado de http://www.nber.org/papers/w12352.pdf.

Ferrante, F. (2005). Revealing Entrepreneurial Talent. Small Business Economics. 25, 159-174.

Fitz-enz, J. (2009). The ROI of Human Capital: measuring the economic value of employee Performance. New York: Amacom.

Goldin, C., \& Katz, L. (1999). The returns to skill in the United States across the twentieth century. Working Paper No. 7126, National Bureau of Economic Research. Recuperado de http://www.nber.org/papers/w7126.pdf

López, J. (2006). La internacionalización de la empresa manufacturera española: Efectos del capital humano genérico y específico. Cuadernos de Gestión, 6(1), 11-24.

Manolova, T., Brush, C., Edelman, L., \& Greene, P. (2002). Internationalization of Small Firms: Personal Factors Revisited. International Small Business Journal, 20(1), 9-31.

Marín, M., Hernández, E., \& Burbano, E. (2018). Impactos del capital humano en las organizaciones empresariales y en los individuos. En Ponencia en el Encuentro Internacional de Investigadores en Administración 2018, Universidad del Valle, Santiago de Cali, Colombia. Recuperado de https://www.academia.edu/38441141/lmpactos_del_Capital_Humano_en_las_Organizaciones_Empresariales_y_en_los_Individuos

Matabanchoy, S. (2012). Salud en el trabajo. Revista Universidad y Salud, 1(15), 87-102.

Mincer, J. (1958). Investment in Human Capital and Personal Income Distribution. The Journal of Political Economy, 66(4), 201-302.

Mungaray, A., \& Ramírez, M. (2007). Capital humano y productividad en microempresas. Investigación Económica, 66(260), 81-115.

Nelson, R., R., \& Phelps, E. S. (1996). Investment in Humans, Technological Diffusion, and Economic Growth. The American Economic Review, 56, 69-75.

Peteraf, M. (1993). The cornerstones of competitive advantage: a resource-based view. Strategic Management Journal, 14(3), 179-191. 
Pucar, S. (2012). The influence of intellectual capital on export performance. Journal of Intellectual Capital, 13(2), 248-261.

Pulic, A. (2004). Intellectual capital - does it create or destroy value? Measuring Business Excellence, 8(1), 62-68. Recuperado de http://dx.doi.org/10.1108/13683040410524757

Say, J. B. (2001). Un tratado de economía política. México D.F.: Fondo de Cultura Económica.

Schultz, T. (1961). Investment in human capital. American Economic Review, 51(1), 1-17.

Siqueira, A. C. (2007). Entrepreneurship and Ethnicity: the Rol of the Human Capital and Family Social Capital. Journal of Developmental Entrepreneurship, 12(1), 31-46.

Smith, A. (2009). Investigación sobre la naturaleza \& causas de la riqueza de las naciones. Madrid: Editorial Tecnos.

Stuart-Mill, J. (2008). Principios de economía política. Madrid: Editorial Síntesis.

Thomas, D. \& E. Frankenberg (2002). Health, Nutrition, and Prosperity: A Microeconomic Perspective. Bulletin of the World Health Organization, 80, 106-113.

Warech, M., \& Tracey, B. (2004). Evaluating the impact of Human Resources: identifying what matters. Cornell Hospitality Quarterly, 45(4), 376-387.

Correo electrónico: eahernandezg@unal.edu.co 